\title{
Phase diagram of Josephson junction arrays with capacitive disorder
}

\author{
F. P. Mancini, P. Sodano, and A. Trombettoni \\ Dipartimento di Fisica and Sezione I.N.F.N., Università di \\ Perugia, Via A. Pascoli, I-06123 Perugia, Italy
}

(October 30, 2018)

\begin{abstract}
We study the phase diagram at finite temperature of Josephson junction arrays with capacitive disorder (i.e., random offset charges and/or random charging energies): in the limit of large particle numbers per junction, this is a remarkable physical realization of the disordered boson Hubbard model. By using a mean-field approximation, we compute the average free energy and the equation for the phase boundary line between the insulating and the superconducting phase. We find that the Mott-insulating lobe structure disappears for large variance $(\sigma \gtrsim e)$ of the offset charges probability distribution. Further, with nearest-neighbor interactions, the insulating lobe around $q=e$ is destroyed even for small values of $\sigma$. In the case of random charging energies, until the variance of the distribution reaches some critical value the superconducting phase increases in comparison to the situation in which all self-capacitances are equal.
\end{abstract}

Typeset using REVTEX 


\section{INTRODUCTION}

In practical realizations of Josephson devices [1], one has to deal with capacitance disorder caused either by offset charge defects in the junctions or in the substrate (random offset charges) [2] or by imperfections in the construction of the devices, which may lead to random capacitances of the Josephson junctions. Random offset charges cannot be made to vanish by using a gate for each superconducting island since in large arrays too many electrodes would be necessary (i.e., too complicated fabrication procedures). In principle, an external uniform charge can be introduced and tuned in Josephson junctions arrays (JJA's) by applying a gate voltage with respect to the ground plane: in Ref. [3] this situation was analyzed experimentally by placing a gate underneath a Josephson array and it was observed a sensible variation $(\sim 40 \%)$ of the resistance between the unfrustrated and the fully frustrated array. Although from a theoretical point of view charge and magnetic frustrations are dual to each other, experimentally it is possible to tune only the magnetic frustration in a controlled way. For this reason, it is widely believed that a challenging task for the theory is to develop reliable techniques to investigate the effects of random charge frustration on the phase structure of the arrays.

In this paper, we shall address the problem of determining the finite temperature phase diagram of JJA's with capacitive disorder (i.e., with random offset charges and/or random self-capacitances). To derive the phase boundary between the insulating and the superconducting phase, we shall use a mean-field (MF) theory approach in the path-integral approach for quantum JJA's with offset charges and general capacitance matrices $[4,5]$. We find that the charge disorder supports superconductivity and that the relative variations of the insulating and superconducting regions depend on the mean value $q$ of the charge probability distribution: when $q=0$, increasing the disorder leads to an enlargement of the superconducting phase. If the charge disorder is sufficiently strong $(\sigma \gtrsim e)$, the lobe structure [1] disappears: in other words, the phase boundary line (and the correlation functions) does not depend any longer on $q$. In the following, we shall provide a quantitative analysis of this phenomenon. We shall consider Gaussian, uniform (as in Ref. [6]) and $\delta$-like distributions. For $T=0$ our results agree with the results obtained in Refs. [6] and [7]. Also the randomness of the self-capacitances leads to remarkable effects: the superconducting phase increases with respect to the case where disorder is not present. We shall also compare the results of the functional MF approach with the molecular MF discussed in Appendix B.

The Hamiltonian commonly used to describe the Cooper pair tunneling in superconducting quantum networks defines the so called quantum phase model (QPM). In its most general form it is given by

$$
H=\frac{1}{2} \sum_{i j}\left(Q_{\mathbf{i}}+q_{\mathbf{i}}\right) C_{\mathbf{i j}}^{-1}\left(Q_{\mathbf{j}}+q_{\mathbf{j}}\right)-E_{J} \sum_{\langle i j\rangle} \cos \left(\varphi_{\mathbf{i}}-\varphi_{\mathbf{j}}\right)
$$

where $\varphi_{\mathbf{i}}$ is the phase of the superconducting order parameter at grain $\mathbf{i}$. Its conjugate variable $n_{\mathbf{i}}\left(\left[\varphi_{\mathbf{i}}, n_{\mathbf{i}}\right]=i \delta_{\mathbf{i j}}\right)$ describes the number of Cooper pairs in the ith superconducting grain. The symbol $\langle i j\rangle$ indicates a sum over nearest-neighbor grains only. The second term in the Hamiltonian (1) describes the hopping of Cooper pairs between neighboring sites $\left(E_{J}\right.$ is the Josephson energy). The first term determines the electrostatic coupling between the Cooper pairs: $Q_{\mathbf{i}}$ is the excess of charge due to Cooper pairs $\left(Q_{\mathbf{i}}=2 e n_{\mathbf{i}}\right)$ on site $\mathbf{i}$ and $C_{\mathbf{i j}}$ is 
the capacitance matrix. An external gate voltage $V_{\mathbf{i}}$ gives the contribution to the energy via the offset charge $q_{\mathbf{i}}=\sum_{\mathbf{j}} C_{\mathbf{i j}} V_{\mathbf{j}}$. This external voltage can be either applied to the ground plane or, more interestingly, it may be induced by charges trapped in the substrate. In the latter situation $q_{\mathbf{i}}$ is a random variable: the effects of this randomness on the phase diagram are the main object of our investigation. We shall also treat explicitly the case of random self-capacitance $C_{\mathbf{i i}}$ which correspond to a random charging energy $E_{C}=e^{2} C_{\mathbf{i i}}^{-1} / 2$.

As is well known [1], the QPM (1) is equivalent to the boson Hubbard model (BHM) in the limit of large particle numbers per junction. The BHM describes soft-core bosons hopping on a lattice [6] and is defined as

$$
H=\frac{1}{2} \sum_{i j} n_{\mathbf{i}} U_{i j} n_{\mathbf{j}}-\mu \sum_{i} n_{\mathbf{i}}-\frac{t}{2} \sum_{\langle i j\rangle}\left(b_{\mathbf{i}}^{\dagger} b_{\mathbf{j}}+H . C .\right) .
$$

Here, $b_{\mathbf{i}}^{\dagger}\left(b_{\mathbf{i}}\right)$ is the creation (annihilation) operator for bosons and $n_{\mathbf{i}}=b_{\mathbf{i}}^{\dagger} b_{\mathbf{i}}$ is the number operator. By writing the field $b_{\mathbf{i}}$ in terms of its amplitude and phase and by neglecting the deviations of the amplitude from its average, we are lead to the QPM (1). An exact mapping between the two models has been derived in Ref. [8]. The hopping term is associated with the Josephson tunneling $\left(\langle n\rangle t \rightarrow E_{J}\right)$ whereas $U_{\mathbf{i j}} \rightarrow 4 e^{2} C_{\mathbf{i j}}^{-1}$ describes the Coulomb interactions between bosons. The chemical potential in the BHM plays a role analogous to the one of the external charge in the QPM $\left(\mu \rightarrow q_{\mathbf{i}}\right)$. Thus a QPM with random offset charge corresponds to a BHM with random on-site energies.

The disordered BHM has attracted much attention during the last decade [6,9-14]. In the pioneering work of Fisher et al. [6] the phase diagram at $T=0$ was studied. Without disorder, the BHM exhibits two types of phases: a superfluid phase and a Mott insulating phase, with the latter characterized by integer (or commensurate) boson densities, by the existence of a gap for the particle-hole excitations, and by zero compressibility. The phase structure is determined by the two competing terms of the Hamiltonian: the charging energy leads to a charge localization in the array, while the Josephson energy induces a phase coherence giving rise to the overall array superfluidity. When disorder is present, a third, intermediate, phase occurs: the Bose glass (BG). This phase has an infinite superfluid susceptibility, but no gap and finite compressibility. It was early realized that considering uniform probability distributions there is no BG in the MF [6]. However, it has been shown that introducing a not uniform triangular probability distribution of offset charges the BG phase appears in the MF phase diagram [9]. A direct Mott insulator to superfluid transition without an intervening $\mathrm{BG}$ phase for weak disorder has been recently investigated in two dimensions $[10,11]$. Very recently, the phase diagram of the two-dimensional disordered BHM has been studied at $T=0$ by means of Monte Carlo simulations [14] evidencing the existence of a Bose glass to superfluid transition in the strong-disorder regime.

In the present paper we shall consider uniform disorder distributions: our MF approach distinguishes the phases with order parameter $\left\langle\cos \varphi_{\mathbf{i}}\right\rangle$ equal to zero (insulating region) or different from zero (superconducting region), but it cannot capture the BG. Therefore, the full phase diagram is much richer than the MF one. It is obvious that for $d=1$ (e.g. JJ chains [15]) the MF fails to provide reliable information. However, the MF allows us to take a first step towards the understanding of the properties of the disordered JJA and BHM at finite temperature. Furthermore, conventional wisdom suggests that for large dimensions the MF approach provides the correct phase diagram of the system. 
The plan of the paper is the following: in Section II, we outline the MF theory for the pure quantum JJA and then we compute the free energy averaged over the disorder. In this way we are able to get a general formula for the phase boundary line at finite temperature. Section III is devoted to the study of the effects of random offset charges with diagonal and nearest-neighbor capacitance matrices. In Section IV we consider random self-capacitances. To check our results, we study in Appendix A the infinite-range hopping limit and provide in Appendix B an alternative and more intuitive MF approach for JJA's in the presence of capacitive disorder. Finally, Sec. V is devoted to concluding remarks.

\section{AVERAGE OVER THE DISORDER IN MEAN-FIELD THEORY}

In a functional approach which makes use of the Hubbard-Stratonovich transformation, the partition function of quantum JJA's may be written as [5]

$$
Z=\int \prod_{\mathbf{i}} D \psi_{\mathbf{i}} D \psi_{\mathbf{i}}^{*} e^{\int_{0}^{\beta} d \tau\left(-\frac{2}{E_{J}} \sum_{\mathbf{i j}} \psi_{\mathbf{i}}^{*} \gamma_{\mathbf{i j}}^{-1} \psi_{\mathbf{j}}\right)} e^{-S_{E f f}[\psi]}
$$

where $\beta=1 / k_{B} T$ and $\gamma_{\mathbf{i j}}=1$ if $\mathbf{i}, \mathbf{j}$ are nearest neighbors and equals zero otherwise (i.e., the hopping term just between nearest neighbors). If $\gamma_{\mathbf{i j}}=1$ for all pairs $\mathbf{i}, \mathbf{j}$ on the lattice, we are led to the infinite-range hopping limit which provides a remarkable example of exactly solvable MF theory [6]. In the following we shall treat explicitly also the infinite-range case. In Eq. (3), $S_{\text {eff }}$ is the effective action for the auxiliary Hubbard-Stratonovich field $\psi_{\mathbf{i}}$ :

$S_{E f f}[\psi]=-\ln \left\{\int \prod_{\mathbf{i}} D \varphi_{\mathbf{i}} \exp \left[\int_{0}^{\beta} d \tau\left(-\frac{1}{2} \sum_{\mathbf{i j}} C_{\mathbf{i} \mathbf{j}} \frac{\dot{\varphi}_{\mathbf{i}}}{2 e} \frac{\dot{\varphi}_{\mathbf{j}}}{2 e}+i \sum_{\mathbf{i}}\left(q_{\mathbf{i}} \frac{\dot{\varphi}_{\mathbf{i}}}{2 e}-\psi_{\mathbf{i}} e^{i \varphi_{\mathbf{i}}}-\psi_{\mathbf{i}}^{*} e^{-i \varphi_{\mathbf{i}}}\right)\right)\right]\right\}$.

The field $\psi_{\mathbf{i}}$ may be regarded as the order parameter for the insulator-superconductor phase transition because it turns out to be proportional to $\left\langle e^{i \varphi_{\mathbf{i}}}\right\rangle$. Since the phase transition is second-order [16], close to the onset of superconductivity the order parameter $\psi_{\mathbf{i}}$ is small. One may then expand the effective action up to the second order in $\psi_{\mathbf{i}}$, getting

$$
Z=\int \prod_{\mathbf{i}} D \psi_{\mathbf{i}} D \psi_{\mathbf{i}}^{*} e^{-F[\psi]}
$$

$F[\psi]$ is the Ginzburg-Landau free energy, which - to the second order in $\psi_{\mathbf{i}}$ - is given by

$$
F[\psi]=\int_{0}^{\beta} d \tau \int_{0}^{\beta} d \tau^{\prime} \sum_{\mathbf{i j}} \psi_{\mathbf{i}}^{*}(\tau)\left[\frac{2}{E_{J}} \gamma_{\mathbf{i j}}^{-1} \delta\left(\tau-\tau^{\prime}\right)-G_{\mathbf{i j}}\left(\tau, \tau^{\prime}\right)\right] \psi_{\mathbf{j}}\left(\tau^{\prime}\right)
$$

where the phase correlator $G_{\mathbf{i j}}$ is given by

$$
G_{\mathbf{i j}}\left(\tau, \tau^{\prime}\right)=\left.\frac{1}{\beta^{2}} \frac{\delta^{2} S_{E f f}[\psi]}{\delta \psi_{\mathbf{i}}(\tau) \delta \psi_{\mathbf{j}}\left(\tau^{\prime}\right)}\right|_{\psi, \psi^{*}=0}=\left\langle e^{i \varphi_{\mathbf{i}}(\tau)-i \varphi_{\mathbf{j}\left(\tau^{\prime}\right)}}\right\rangle_{0}
$$

A straightforward, but lengthy, calculation gives [5] 


$$
\begin{gathered}
G_{\mathbf{r s}}\left(\tau ; \tau^{\prime}\right)=\delta_{\mathbf{r s}} e^{-2 e^{2} C_{\mathbf{r r}}^{-1}\left|\tau-\tau^{\prime}\right|} . \\
\cdot \frac{\sum_{\left[n_{\mathbf{i}}\right]} e^{-\sum_{\mathbf{i j}} 2 e^{2} \beta C_{\mathbf{i j}}^{-1}\left(n_{\mathbf{i}}+\frac{q_{\mathbf{i}}}{2 e}\right)\left(n_{\mathbf{j}}+\frac{q_{\mathbf{j}}}{2 e}\right)-\sum_{\mathbf{i}} 4 e^{2} C_{\mathbf{r i}}^{-1}\left(n_{\mathbf{i}}+\frac{q_{\mathbf{i}}}{2 e}\right)\left(\tau-\tau^{\prime}\right)}}{\sum_{\left[n_{\mathbf{i}}\right]} e^{-\sum_{\mathbf{i} \mathbf{j}} 2 \beta e^{2} C_{\mathbf{i} \mathbf{j}}^{-1}\left(n_{\mathbf{i}}+\frac{q_{\mathbf{i}}}{2 e}\right)\left(n_{\mathbf{j}}+\frac{q_{\mathbf{j}}}{2 e}\right)}} .
\end{gathered}
$$

Here, $n_{\mathbf{i}}$ assumes all integer values and $\sum_{\left[n_{\mathbf{i}}\right]}$ is a sum over all the configurations. If one introduces the Fourier transforms in the imaginary time and in the space

$$
\begin{aligned}
\psi_{\mathbf{i}}(\tau) & =\frac{1}{\beta N} \sum_{\mathbf{k} \mu} \psi_{\mathbf{k}}\left(\omega_{\mu}\right) e^{i\left(\mathbf{k} \cdot \mathbf{i}+\omega_{\mu} \tau\right)}, \\
G_{\mathbf{i}}(\tau) & =\frac{1}{\beta N} \sum_{\mathbf{k} \mu} G_{\mathbf{k}}\left(\omega_{\mu}\right) e^{i\left(\mathbf{k} \cdot \mathbf{i}+\omega_{\mu} \tau\right)}
\end{aligned}
$$

(with $\omega_{\mu}$ Bose-Matsubara frequencies and $\mathbf{k}$ vectors of the reciprocal lattice), the GinzburgLandau free energy $(6)$, reads $[7,17]$

$$
F[\psi]=\frac{1}{\beta N} \sum_{\mu \mathbf{k} \mathbf{k}^{\prime}} \psi_{\mathbf{k}}^{*}\left(\omega_{\mu}\right)\left[\frac{2}{E_{J}} \gamma_{\mathbf{k}}^{-1} \delta_{\mathbf{k k}^{\prime}}-\frac{G_{\mathbf{k}-\mathbf{k}^{\prime}}\left(\omega_{\mu}\right)}{N}\right] \psi_{\mathbf{k}^{\prime}}\left(\omega_{\mu}\right),
$$

where

$$
\gamma_{\mathbf{i j}}^{-1}=\frac{1}{N} \sum_{\mathbf{k}} \gamma_{\mathbf{k}}^{-1} e^{i \mathbf{k} \cdot(\mathbf{i}-\mathbf{j})}
$$

$\gamma_{\mathbf{k}}^{-1}$ is the inverse of the Fourier transform of the Josephson coupling strength $\gamma_{\mathbf{i j}}$; since $\gamma_{\mathbf{i j}}=1$ if $\mathbf{i}, \mathbf{j}$ are nearest neighbors and zero otherwise, we have $\gamma_{\mathbf{k}}^{-1}=\left(\sum_{\mathbf{p}} e^{-i \mathbf{k} \cdot \mathbf{p}}\right)^{-1}$, where $\mathbf{p}$ is a vector connecting two nearest-neighbors sites. Expanding in $\mathbf{k}$ one gets $\gamma_{\mathbf{k}}^{-1}=1 / z+\cdots$, where $z$ is the coordination number. Substituting in Eq. (11) and keeping only the lowestorder terms in $\omega_{\mu}, \mathbf{k}$ and $1 / z$, the MF Ginzburg-Landau free energy reads

$$
F[\psi] \simeq \frac{1}{\beta N} \sum_{\mathbf{k} \mu}\left[\frac{2}{E_{J} z}-G_{\mathbf{0}}+\cdots\right]\left|\psi_{\mathbf{k}}\left(\omega_{\mu}\right)\right|^{2} .
$$

In Eq. (13), $G_{0}$ is

$$
G_{\mathbf{0}}=\frac{1}{N} \sum_{\mathbf{r}} G_{\mathbf{r}}\left(\omega_{\mu}=0, T=T_{c}\right) .
$$

As evidenced in Ref. [5] the MF theory approximation amounts to neglect all higher order terms in Eq. (13).

For a given realization of the disorder, the Ginzburg-Landau free energy (i.e., the free energy near the transition) is given by Eq. (13). The average of the free energy over all the possible realization of the disorder allows for evaluation of the effect of a random charge frustration $\left\{q_{\mathbf{i}}\right\}$ or a random diagonal charging energy $U_{\mathbf{i i}}=4 e^{2} C_{\mathbf{i i}}^{-1}$ : one has then

$$
\bar{F}[\psi]=\int d\{X\} P(\{X\}) F[\psi],
$$


where $P(\{X\})$ is a given probability distribution and $d\{X\} P(\{X\})=\prod_{\mathbf{i}} d q_{\mathbf{i}} P\left(q_{\mathbf{i}}\right)$ when one considers random offset charges or $d\{X\} P(\{X\})=\prod_{\mathbf{i}} d U_{\mathbf{i i}} P\left(U_{\mathbf{i i}}\right)$ for random charging energies. The random variables on different sites are taken to be independent. The phase boundary line between the insulating and the superconducting phase is determined by requiring that $\bar{F}=0$, which in turn leads to

$$
1=z \frac{E_{J}}{2} \bar{G}_{\mathbf{0}}
$$

In Appendix A we will discuss how Eq. (16) is modified in the infinite-range hopping limit. A comparison of the MF discussed in this Section with an alternative MF approach in the presence of disorder is given in Appendix B.

\section{RANDOM OFFSET CHARGES}

In the following, we shall consider three different random offset charges probability distribution with mean $q$ and width $\sigma$. That is, a Gaussian distribution $P\left(q_{\mathbf{i}}\right)=$ const $\times \cdot e^{-\left(q_{\mathbf{i}}-q\right)^{2} / 2 \sigma^{2}}$, a uniform distribution $P\left(q_{\mathbf{i}}\right)=$ const between $q-\sigma$, and $q+\sigma$ and a sum of $\delta$-like distributions $P\left(q_{\mathbf{i}}\right)=\sum_{n} p_{n} \delta\left(q_{\mathbf{i}}-n e\right)$, with $\sum_{n} p_{n}=1$. For a diagonal capacitance matrix, Eq. (16) leads to

$$
\frac{1}{\alpha}=\int d q P(q) g(q, y)
$$

with $\alpha=z E_{J} / 4 E_{c}, y=k_{B} T_{c} / E_{c}$, and

$$
g(q, y)=\frac{\sum_{n} e^{-\frac{4}{y}(n+q / 2 e)^{2}} \frac{1}{1-4(n+q / 2 e)^{2}}}{\sum_{m} e^{-\frac{4}{y}(m+q / 2 e)^{2}}} .
$$

We observe that the lobes of JJA are invariant under $q / 2 e \rightarrow q / 2 e+1$ and symmetric around $q / 2 e=n+1 / 2$, where $n$ is an integer:

$$
g(q+2 n e, y)=g(q, y) ; g(n+1 / 2+q / 2 e, y)=g(n+1 / 2-q / 2 e, y) .
$$

If one considers the infinite-range hopping limit, one still gets Eq. (17) provided that $\alpha=J / 4 E_{c}$.

The results obtained from Eq. (17) with a Gaussian distribution are displayed in Fig. 1. One observes that when $q / 2 e=0$, increasing $\sigma$ favors the superconducting phase while, when $q / 2 e=1 / 2$, increasing $\sigma$ leads to the increase of the insulating phase. For large $\sigma$ (i.e., $\sigma \gtrsim e$ ), the phase boundary line is the same for all the values of $q$ (in Fig. 1 the large- $\sigma$ behavior is represented by the bold line). This is expected since, when $\sigma$ is large, the average free energy $\bar{F}$ does not depend any longer on $q$.

A useful representation of the phase diagram is obtained by plotting, at fixed temperature, the phase boundary line on the plane $q$ - $\alpha$. Without disorder one observes the well-known lobe structure [1]. In the presence of weak disorder and at $T=0$, the lobes shrink, evidencing a decrease of the insulating phase: for a Gaussian (or unbounded) distribution the insulating phase completely disappears even for an arbitrarily weak disorder 
[6]. In Fig. 2 we exhibit the phase boundary line on the plane $q-\alpha$ at finite temperature for the Gaussian and uniform distributions. Of course, even at finite temperature, when the disorder increases the lobes flatten and the same lobe structure is obtained from both distributions. At $T=0$ we recover the result of Ref. [6]. This can be easily seen if one observes that, at low temperatures, for $|q|<e$, one has [7]

$$
g(q, y \rightarrow 0)=\frac{1}{1-4\left(\frac{q}{2 e}\right)^{2}} .
$$

Without disorder $(\sigma=0)$, Eq. (17) simply gives $\alpha=1-4(q / 2 e)^{2}$. With the Gaussian distribution, since $g$ has a pole in the half-integer value of the Cooper charge, the integral in Eq. (17) diverges, and $\alpha \rightarrow 0$; i.e., the lobes disappear for every value of $\sigma$. As evidenced in Fig. 3, for a uniform distribution, when $\sigma>e$, then $\alpha \rightarrow 0$; when $\sigma<e, \alpha \rightarrow 0$ only for $e-\sigma \leq q \leq e+\sigma$ in agreement with [6].

We now consider the case

$$
P\left(q_{\mathbf{i}}\right)=\sum_{n} p_{n} \delta\left(q_{\mathbf{i}}-n e\right),
$$

with $\sum_{n} p_{n}=1$, corresponding to a random distribution of charges which are integer multiples of $e$. Actually, this is the most realistic situation for a random distribution. Indeed, the probability distributions employed before should be viewed as fictitious continuous distributions; i.e., the properties of the overall distribution of charges (mean value and width) can be well approximated with a continuous distribution $P(q)$. Inserting the probability distribution (21) in (18) we have

$$
\int d q P(q) g(q, y)=\int d q \sum_{n} p_{n} \delta(q-n e) g(q, y)=\sum_{o d d} p_{n} g(n e, y)+\sum_{e v e n} p_{n} g(n e, y)
$$

where $\sum_{\text {odd }}\left(\sum_{\text {even }}\right)$ is a sum restricted to odd (even) integer. Recalling Eq. (19), we have $g(2 n e, y)=g(0, y)$ and $g((2 n+1) e, y)=g(e, y)$, and we find

$$
\frac{1}{\alpha}=p_{0} g(0, y)+p_{e} g(e, y)
$$

where $p_{0}=\sum_{\text {even }} p_{n}\left(p_{e}=\sum_{\text {odd }} p_{n}\right)$ is the probability that the offset charge $q$ is an even (odd) integer multiple of $e$. In Fig. 4 we plot the phase boundary line (22) for $p_{0}=p_{e}=1 / 2$.

We are able to show now that applying the MF approximation described in Appendix B with the probability distribution (21) it is possible to find exactly Eq. (22). The eigenvalue equation for the Hamiltonian (B1), $H_{\mathbf{i}} \psi_{n}=E_{n} \psi_{n}$, can be recast in the standard form of the Mathieu equation with the transformation $\psi_{n}\left(\varphi_{\mathbf{i}}\right)=e^{-i \frac{q_{\mathbf{i}}}{2 e} \varphi_{\mathbf{i}}} \rho_{n}\left(\varphi_{\mathbf{i}}\right)$. We find

$$
\frac{d^{2} \rho_{n}}{d \varphi_{\mathbf{i}}^{2}}+\left(\frac{\lambda_{n}}{4}-\frac{v}{2} \cos \varphi\right) \rho_{n}=0
$$

where $\lambda_{n}=E_{n} / E_{C}$ and $v=-z E_{J} \overline{\langle\cos \varphi\rangle} / 2 E_{C}$. The periodic boundary condition $\psi_{n}\left(\varphi_{\mathbf{i}}\right)=$ $\psi_{n}\left(\varphi_{\mathbf{i}}+2 \pi\right)$ gives $\rho_{n}\left(\varphi_{\mathbf{i}}\right)=\rho_{n}\left(\varphi_{\mathbf{i}}+2 \pi\right)$ for $q_{\mathbf{i}}=2$ ne and $\rho_{n}\left(\varphi_{\mathbf{i}}\right)=-\rho_{n}\left(\varphi_{\mathbf{i}}+2 \pi\right)$ for $q_{\mathbf{i}}=$ $2(n+1) e(n$ integer $)$. For small values of $v$, the periodic and antiperiodic solutions of the Mathieu equation can be calculated analytically, giving exactly Eq. (22). 


\section{A. NONDIAGONAL CAPACITANCE MATRICES}

With nondiagonal capacitance matrices, the phase diagram without disorder becomes richer [1]. For concreteness, we shall consider on-site and a weaker nearest-neighbor (NN) interaction; i.e., the inverse capacitance matrix is restricted to diagonal and $\mathrm{NN}$ terms. If one defines $\theta$ as the ratio between $\mathrm{NN}$ and diagonal terms, one should restrict only to $z \theta<1$ in order to insure the invertibility of the capacitance matrix [18]. Without disorder, at $T=0$ an insulating lobe around $q=e$ appears: the width of this lobe is $z \theta /(1+z \theta)$. Putting $W=1+z \theta$, Eq. (18) for $|q / 2 e|<1 / 2 W$ gives $g(q, y \rightarrow 0)=1 /\left[1-4 W^{2}(q / 2 e)^{2}\right]$; for $1 / 2 W<q / 2 e<1-(1 / 2 W)$ it becomes [7]

$$
g(q, y \rightarrow 0)=-\frac{1}{2}\left[\frac{1}{\left(2 W \frac{q}{2 e}-1\right)\left(2 W \frac{q}{2 e}-3\right)}+\frac{1}{\left(2 W\left(\frac{q}{2 e}-1\right)+1\right)\left(2 W\left(\frac{q}{2 e}-1\right)+3\right)}\right] .
$$

In presence of disorder, Eq. (17) for a uniform distribution gives $\alpha=0$ for $(1 / 2 W)-\sigma \leq q \leq$ $(1 / 2 W)+\sigma$ and $1-(1 / 2 W)-\sigma \leq q \leq 1-(1 / 2 W)+\sigma$. Thus, the lobe width decreases as $(z \theta-2 \sigma W) / W$. One sees that for $\sigma=z \theta / 2 W$ the insulating lobe around $q=e$ disappears. This phenomenon is evidenced in Fig. 5 .

\section{RANDOM SELF-CAPACITANCES}

In the following we limit our analysis only to JJA's with random self-capacitance $C_{\mathbf{i i}}$ and uniform charge frustration $q$. We shall consider, in fact, a random diagonal charging energy $U_{\text {ii }}$, which we assume to be independently distributed according to the probability distribution $P\left(U_{\mathbf{i i}}\right) \propto e^{-\left(U_{\mathbf{i i}}-U_{0}\right)^{2} / 2 \sigma^{2}}$, where the diagonal electrostatic contribution to the energy $U_{\text {ii }}$ needs to be positive. By averaging the free energy (15), the equation for the phase boundary becomes

$$
\frac{1}{\alpha}=\int_{0}^{\infty} d U \frac{P(U)}{U} g(U, y)
$$

where now $\alpha=z E_{J} / 4 U_{0}$ and the function $g(U, y)$ is given by

$$
g(U, y)=\frac{\sum_{n} e^{-\frac{4}{y} U(n+q / 2 e)^{2}} \frac{1}{1-4(n+q / 2 e)^{2}}}{\sum_{m} e^{-\frac{4}{y} U(m+q / 2 e)^{2}}} .
$$

The results of Eq. (24) are summarized in Figs. 6 and 7: when $\sigma$ is small, the superconducting phase increases in comparison to the nonrandom case: this is due to the factor $1 / U$ in Eq. (24), which makes larger the contribution of junctions with charging energies less than $U_{0}$. The increase of the superconducting phase is thus due to a decrease of the effective value of $E_{c}$. This behavior occurs until $\sigma$ reaches a critical value (depending on the charge frustration and on the temperature), of order $U_{0}$ : at this value of $\sigma$ the insulating region starts to increase. This is due to the asymmetry of the distribution, which has its peak in $U_{0}$, but only for positive values. This phenomenon is present also if one considers different distributions. An interesting observation is that, when $q / 2 e=1 / 2$ (maximum frustration induced by the external offset charges), the randomness does not modify considerably the phase diagram. This should be compared with the nonfrustrated case $(q / 2 e=0)$, where randomness sensibly affects the phase diagram. 


\section{CONCLUSIONS}

We obtained the phase diagram at finite temperature of JJA's with capacitive disorder (i.e., random offset charges and/or random charging energies) by using a MF approximation. For a random distribution of offset charges with mean $q$ and variance $\sigma$, one has that for $\sigma \gtrsim e$, the phase boundary line coincides for any value of $q$ and the lobe structures on the plane $q-\alpha$ disappear ( $\alpha$ is the ratio between the Josephson and charging energies). At $T=0$ the result of Ref. [6] are retrieved. If one considers in the BHM also a nearestneighbor interaction, the insulating lobe around $q=e$, which arises in absence of disorder, is destroyed even for small values of $\sigma$. For arrays with random charging energies, when the variance of the probability distribution is smaller than a critical value, the superconducting phase increases with respect to the situation in which all self-capacitances are equal. To check our results, we have considered also the infinite-range hopping limit of the QPM.

Within the MF approach used here, it is not possible to capture the Bose glass phase and therefore the full physical picture can be much richer than the one extracted from MF. However, if in low-dimensional systems (e.g., in Josephson junction chains) the MF is expected to fail [15], we envisage that our results provide qualitatively correct predictions in large dimensional arrays.

Acknowledgements We thank A.R. Bishop, F. Cooper, G. Grignani, A. Mattoni, A. Smerzi, and A. Tagliacozzo for interesting and fruitful discussions. A.T. and P.S. are grateful to the Los Alamos National Laboratory for the kind hospitality and for partial financial support in the final stage of this work. This research has been financed by M.I.U.R. under grant No. 2001028294.

\section{APPENDIX A: INFINITE-RANGE HOPPING LIMIT}

We consider the Hamiltonian (1) in the limit of infinite-range hopping, i.e., $\gamma_{\mathbf{i j}}=1$ if $\mathbf{i} \neq \mathbf{j}$ and $\gamma_{\mathbf{i j}}=0$ if $\mathbf{i}=\mathbf{j}$. A meaningful thermodynamic limit $(N \rightarrow \infty)$ is ensured by the scaling of the Josephson term $E_{J} \equiv J / N$. This model has been studied in Ref. [6] for the disordered BHM at $T=0$. Here we study the finite temperature case within the approach

provided in this paper. From $\gamma_{\mathbf{i j}}=\frac{1}{N} \sum_{\mathbf{k}} \gamma_{\mathbf{k}} e^{i \mathbf{k} \cdot(\mathbf{i}-\mathbf{j})}$, it follows that $\gamma_{\mathbf{k}}=N \delta_{\mathbf{k}, 0}-1$. Therefore the free energy (11) is

$$
F[\psi]=\frac{1}{\beta N} \sum_{\mu \mathbf{k} \mathbf{k}^{\prime}} \psi_{\mathbf{k}}^{*}\left(\omega_{\mu}\right)\left[\frac{2 N \delta_{\mathbf{k k}^{\prime}}}{J\left(N \delta_{\mathbf{k}, 0}-1\right)}-\frac{G_{\mathbf{k}-\mathbf{k}^{\prime}}\left(\omega_{\mu}\right)}{N}\right] \psi_{\mathbf{k}^{\prime}}\left(\omega_{\mu}\right) .
$$

Averaging over the disorder, requiring $\bar{F}=0$, for $N \rightarrow \infty$ one has

$$
1=\frac{J}{2} \bar{G}_{0}
$$

A comparison with Eq. (16) makes evident that the results of Secs. III and IV apply also in the infinite-range hopping model, provided that $z E_{J} \rightarrow J$. 


\section{APPENDIX B: AN ALTERNATIVE MEAN-FIELD APPROACH}

The effect of the quantum fluctuations is generally underestimated in MF theory: we expect that a MF theory approach could provide qualitative information on the phase diagram in three dimensions, but not in lower dimensions. In fact, a similar situation arises in quantum spin glasses where MF treatments are able to establish the existence of a spin glass transition [19]. Even if powerful and elegant, the mean-field approximation in the functional approach should be regarded only as a first step in understanding the role played by the disorder in the mean-field average which leads to Eq. (16).

The aim of this appendix is to make more explicit the relationship between the average over the disorder and the quantum statistical average by resorting to a molecular field theory approach. In fact, we shall present an alternative and more intuitive mean-field approach, which may be easily applied only to JJA's with diagonal capacitance matrices.

For this purpose, let us consider a JJA's with a fixed charging energy $E_{C}$ and a random distribution of offset charges $P\left(q_{\mathbf{i}}\right)$. The charging term in the QPM Hamiltonian (1) is then diagonal and the Josephson term couples different sites. Mean-field theory consists in replacing the Josephson coupling of the phase on a given island $\mathbf{i}$ to its neighbors by an average coupling: $E_{J} \sum_{\langle\mathbf{i} j\rangle} \cos \left(\varphi_{\mathbf{i}}-\varphi_{\mathbf{j}}\right)=z E_{J} \overline{\langle\cos \varphi\rangle} \sum_{\mathbf{i}} \cos \varphi_{\mathbf{i}}$. In this way the Hamiltonian becomes a sum of single site Hamiltonians, $H=\sum_{\mathbf{i}} H_{\mathbf{i}}$, where

$$
H_{\mathbf{i}}=-4 E_{C} \frac{\partial^{2}}{\partial \varphi_{\mathbf{i}}^{2}}-8 i E_{C} \frac{q_{\mathbf{i}}}{2 e} \frac{\partial}{\partial \varphi_{\mathbf{i}}}-z E_{J} \overline{\langle\cos \varphi\rangle} \cos \varphi_{\mathbf{i}}
$$

The single-site Hamiltonian (B1) depends on the random charge frustration $q_{\mathrm{i}}$ : thus, its eigenfunctions and eigenvalues depend on $q_{\mathbf{i}}$. Therefore, one has to impose the selfconsistency condition with a double average, the quantum one and the average over the disorder:

$$
\overline{\langle\cos \varphi\rangle}=\int d q_{\mathbf{i}} P\left(q_{\mathbf{i}}\right) \frac{\sum_{n} e^{-\beta E_{n}}\left\langle\psi_{n}|\cos \varphi| \psi_{n}\right\rangle}{\sum_{n} e^{-\beta E_{n}}}
$$

where the $\psi_{n}$ are the eigenfunctions of the single-site Hamiltonian $H_{i}$. The phase boundary line is obtained from Eq. (B2) by requiring $\overline{\langle\cos \varphi\rangle}$ to be small and by keeping only terms proportional to it (we recall that the transition is second order).

We conclude this appendix by stressing that the application of mean-field theory in the presence of disorder corresponds to introducing an order parameter, which is averaged also over the disorder: the self-consistency condition then gives the correct mean-field phase boundary line. In Sec. III, Eqs. (B2) and (16) are compared for the $\delta$-like probability distribution, showing that they give the same results. 


\section{REFERENCES}

[1] For a review see R. Fazio and H. van der Zant, Phys. Rep. 355, 235 (2001), and references therein.

[2] V.A. Krupenin, D.E. Presnov, A.B. Zorin, and J. Niemeyer, J. Low Temp. Phys. 118, $287(2000)$.

[3] P. Lafarge, J.J. Meindersma, and J.E. Mooji, in Macroscopic Quantum Phenomena and Coherence in Superconducting Networks, edited by C. Giovanella and M. Tinkham (World Scientific, Singapore, 1995), p. 94.

[4] T.K. Kopeć and J.V. José, Phys. Rev. Lett. 84, 749 (2000).

[5] G. Grignani, A. Mattoni, P. Sodano, and A. Trombettoni, Phys. Rev. B 61, 11676 (2000).

[6] M.P.A. Fisher, P.B. Weichman, G. Grinstein, and D.S. Fisher, Phys. Rev. B 40, 546 (1989).

[7] A. van Otterlo, K.-H. Wagenblast, R. Fazio and G. Schön, Phys. Rev. B 48, 3316 (1993).

[8] J.R. Anglin, P. Drummond, and A. Smerzi, Phys. Rev. A 64, 063605 (2001).

[9] F. Pázmándi, G. Zimányi, and R. Scalettar, Phys. Rev. Lett. 75, 1356 (1995); Europhys. Lett. 38, 255 (1997).

[10] J. Kisker and H. Rieger, Phys. Rev. B 55, 11981 (1997).

[11] F. Pázmándi and G.T. Zimányi, Phys. Rev. B 57, 5044 (1998).

[12] I.F. Herbut, Phys. Rev. B 57, 13729 (1998).

[13] E. Granato and J.M. Kosterlitz, Phys. Rev. Lett. 81, 3888 (1998).

[14] J.-W. Lee, M.-C. Cha, and D. Kim, Phys. Rev. Lett. 87, 247006 (2001); 88, 049901 (2002).

[15] P.A. Bobbert, R. Fazio, G. Schön, and A.D. Zaikin, Phys. Rev. B 45, 2294 (1992).

[16] M.V. Simkin, Physica C 267, 161 (1996).

[17] S.V. Panyukov and A.D. Zaikin, J. Low Temp. Phys. 75, 361 (1989).

[18] R.S. Fishman and D. Stroud, Phys. Rev. B 37, 1499 (1988).

[19] A.J. Bray and M.A. Moore, J. Phys. C 13, L655 (1980). 


\section{FIGURES}

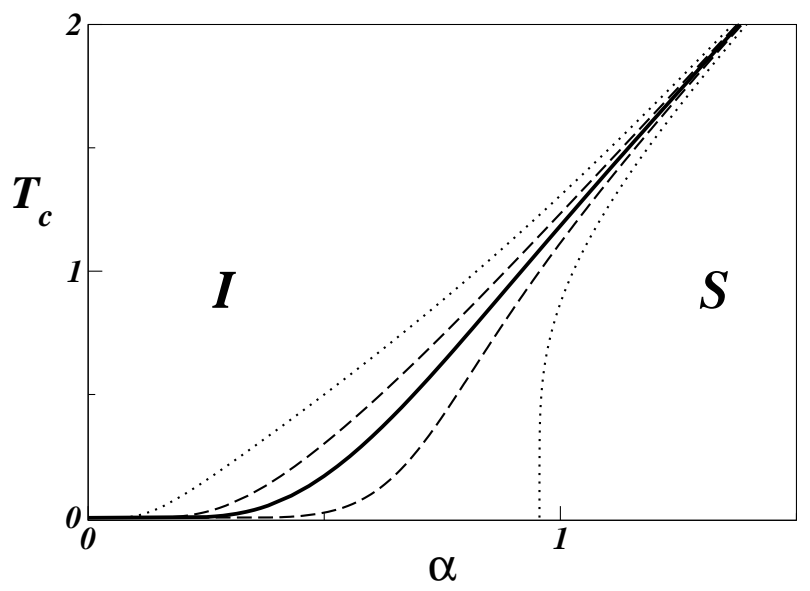

FIG. 1. Phase diagram for random offset charges with Gaussian distribution ( $T_{c}$ is in units of $\left.k_{B} / E_{C}\right)$. The bold line is for $\sigma=e$ and it represents the case of large variance and it is found for all values of the mean $q$. To the left (right), we plot $q=e(q=0)$; we use $\sigma / 2 e=0.1$ (dotted line) and 0.25 (dashed line). The $\mathbf{I}$ and $\mathbf{S}$ indicate, respectively, insulating and superconducting phase.

(a)

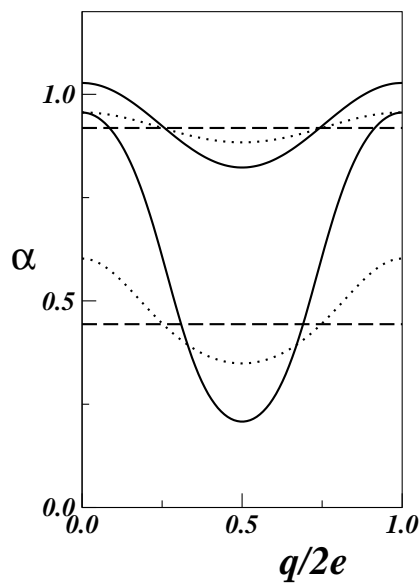

(b)

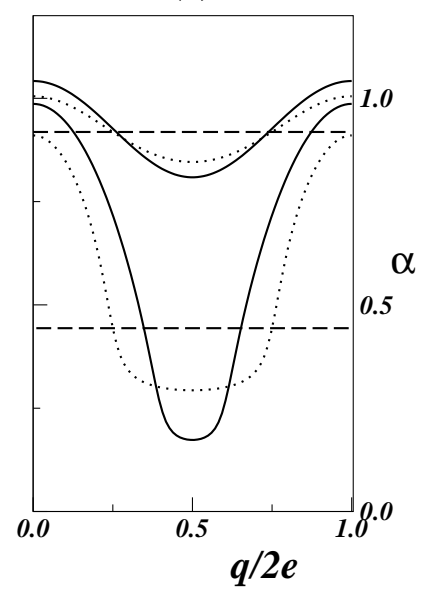

FIG. 2. Phase diagram with diagonal capacitance and random offset charges with Gaussian (a) and uniform (b) distribution. Top (bottom) of the figures: $k_{B} T / E_{C}=1(0.1)$. We plot the cases $\sigma / 2 e=0.1$ (solid lines), 0.25 (dotted lines), 0.5 (dashed lines). For large $\sigma / 2 e$ the phase boundary line is flat and it is the same for both distributions. 


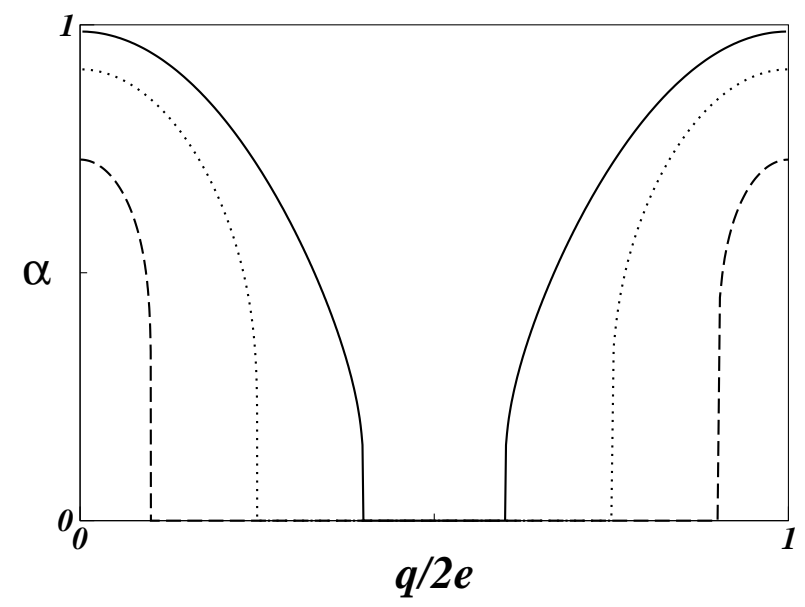

FIG. 3. Phase diagram at $T=0$ for random offset charges with uniform distribution and short-ranged inverse capacitance matrix. $\sigma / 2 e$ is respectively 0.1 (solid line), 0.25 (dotted line) and 0.40 (dashed line).

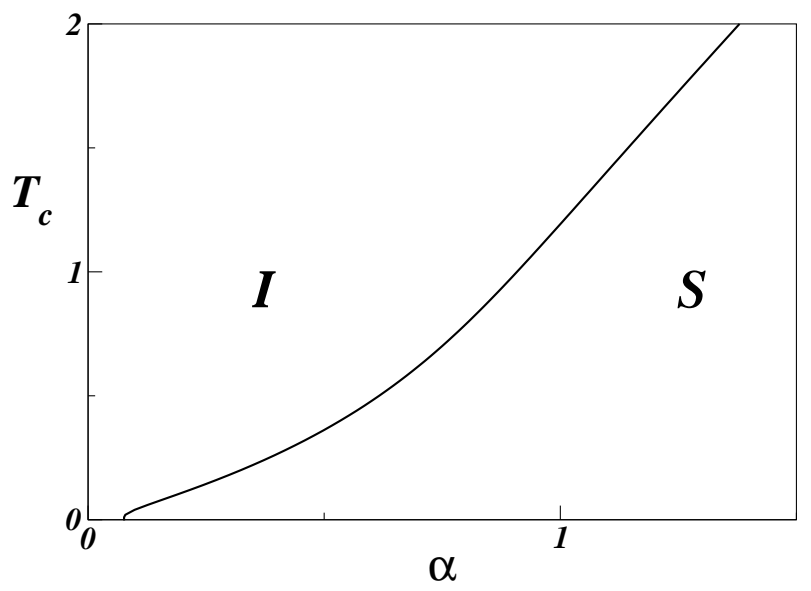

FIG. 4. Phase diagram for random offset charges with probability distribution given by (21) and short-ranged inverse capacitance matrix. In the plot $p_{0}=p_{e}=1 / 2$. 


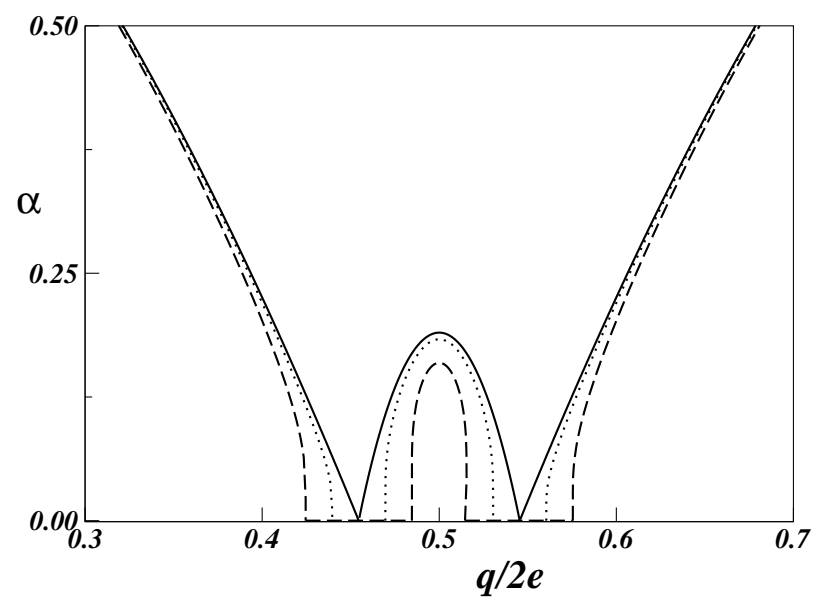

FIG. 5. Phase diagram at $T=0$ for random offset charges with uniform distribution and short-ranged inverse capacitance matrix. In the plot $z \theta$ is equal to 0.1 while $\sigma / 2 e$ is respectively 0 (solid line), 0.015 (dotted line) and 0.03 (dashed line). For this value of $z \theta$, the lobe disappears at $\sigma=0.045$.

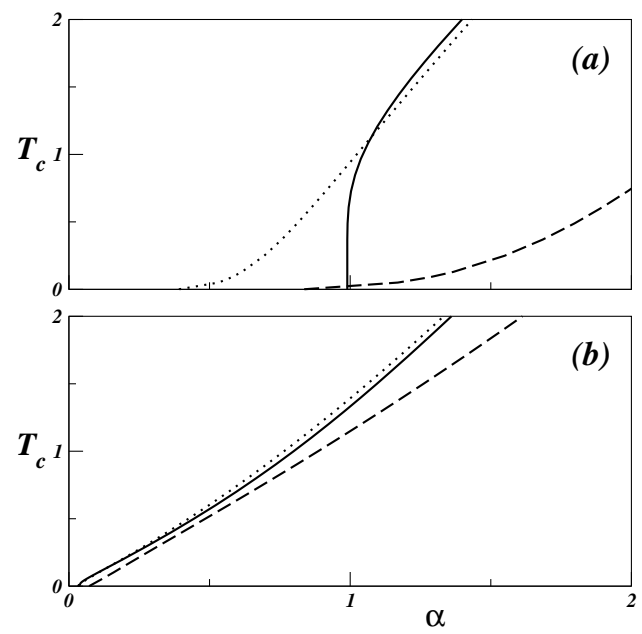

FIG. 6. Phase diagram in the $T_{c}-\alpha$ plane for random diagonal capacitance with Gaussian distribution and uniform offset charge $q / 2 e=0$ (a), 0.5 (b) ( $T_{c}$ is in units of $\left.k_{B} / U_{0}\right)$ while $\sigma / 2 e$ is 0.1 (solid line), 1 (dotted line) and 5 (dashed line). 


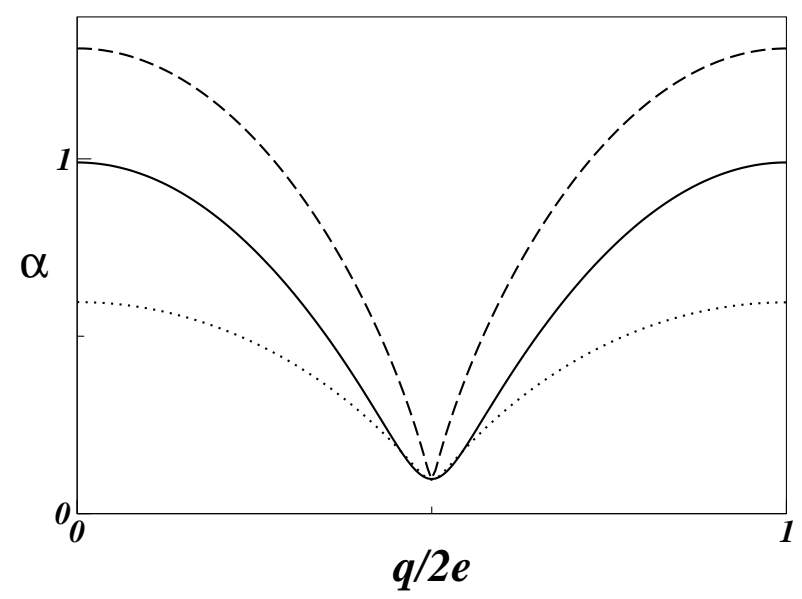

FIG. 7. Phase diagram in the $q-\alpha$ plane for random diagonal capacitance with Gaussian distribution and uniform offset charge at $k_{B} T / U_{0}=0.1 . \sigma / 2 e=0.1$ (solid line), 1 (dotted line) and 5 (dashed line). 